\title{
Two-loop binding corrections to the electron gyromagnetic factor
}

\author{
Andrzej Czarnecki, ${ }^{1}$ Matthew Dowling, ${ }^{1}$ Jan Piclum,,${ }^{1,2}$ and Robert Szafron ${ }^{1,3}$ \\ ${ }^{1}$ Department of Physics, University of Alberta, Edmonton, Alberta, Canada T6G 2E1 \\ ${ }^{2}$ Theoretische Physik 1, Naturwissenschaftlich-Technische Fakultät, Universität Siegen, 57068 Siegen, Germany \\ ${ }^{3}$ Physik Department T31, Technische Universität München, \\ James Franck Straße 1, 85748 Garching, Germany
}

\begin{abstract}
We compute corrections to the gyromagnetic factor of an electron bound in a hydrogen-like ion at order $\alpha^{2}(Z \alpha)^{5}$. This result removes a major uncertainty in predictions for silicon and carbon ions, used to determine the atomic mass of the electron.
\end{abstract}

PACS numbers: 31.30.js, 06.20.Jr, 31.30.jc

The Dirac equation predicts the gyromagnetic factor of a point-like electron to be $g=2$. However, even in vacuum, self-interaction of the electron modifies its $g$. The deviation from Dirac's prediction, a dimensionless number known as $g-2$, can be computed in quantum electrodynamics (QED) and expressed as a perturbation series in the fine-structure constant $\alpha=1 / 137.035999139(31)$ [1]. This research program earned the 1965 Nobel Prize [2] and has led to the stunning five-loop prediction of $\operatorname{order}\left(\frac{\alpha}{\pi}\right)^{5}[3]$. In another heroic development, the fourloop term has been calculated with very high precision [4]. On the experimental side, precision is so high that the electron $g-2$ is currently the best source of $\alpha[5]$.

When the electron is bound in an atom, the presence of the electrically-charged nucleus also influences the $g$ factor. This matters greatly for studies of trapped ions. The $g$-factor has been precisely measured in a range of hydrogen-, lithium- and boron-like ions [6 13]. This experimental enterprise holds great potential for the determination of fundamental constants and tests of the Standard Model because of the variety of systems that can be measured [14]. Access to nuclei with diverse values of the atomic number $Z$, and multiple electronic configurations for a given $Z$, helps eliminate uncertainties. Already now the hydrogen-like carbon (combined with silicon) provides the most precise value of the electron mass [15, 16]. In the future, also the fine-structure constant might be determined independently from the theory and measurements of the free-electron $g-2$ [17 19$]$.

The leading binding effect on $g$ in a hydrogen-like ion, related to the electron's motion, is known to all orders in Coulomb interactions between the electron and the nucleus [20]; with $\alpha_{Z}=Z \alpha$,

$$
g^{(0)}=2-\frac{4}{3}\left(1-\sqrt{1-\alpha_{Z}^{2}}\right) .
$$

In our notation $g^{(a, b)}, a$ denotes the power of $\frac{\alpha}{\pi}$ and $b$, if present, the power of $\alpha_{Z}$. Considered together, selfinteraction and binding effects are described by a double expansion in $\frac{\alpha}{\pi}$ and $\alpha_{Z}$.

At one-loop level of self-interaction, $g$ is known analytically including the very recently computed terms of

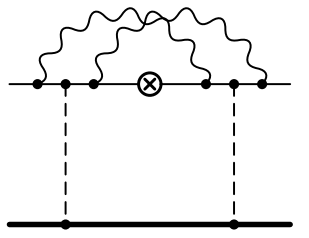

(SE)

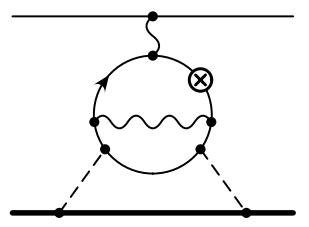

$(\mathrm{MLPH})$

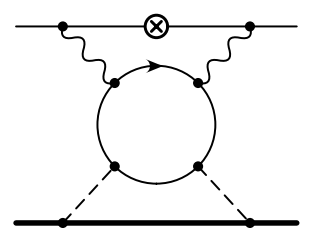

$(\mathrm{LBL})$

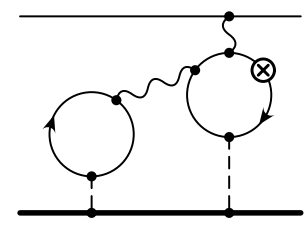

(MLVP)
FIG. 1. Examples of new contributions to the $g$-factor at order $\alpha^{2} \alpha_{Z}^{5}$. Coupling of the external magnetic field is denoted with a circled cross. (SE) one of about 100 two-loop selfenergy diagrams; (LBL) light-by-light scattering; (MLPH), (MLVP) corrections to the so-called magnetic loop with a virtual photon and a vacuum polarization.

order $\frac{\alpha}{\pi} \alpha_{Z}^{5}$ [21]. Higher order terms in $\alpha_{Z}$ have been computed numerically [22]. Numerical methods work especially well for highly-charged ions but struggle for low values of $Z$. On the other hand, the perturbative series in $\alpha_{Z}$ behaves best at small $Z$. Thus analytical and numerical methods are complementary.

There is a slight tension between the two approaches. Corrections of $\mathcal{O}\left(\frac{\alpha}{\pi} \alpha_{Z}^{5}\right)$ arise from diagrams similar to Fig. 11(SE), with a single self-energy (SE) loop. For an $n \mathrm{~S}$ state ( $n$ is the principal quantum number) they are

$$
g^{(1,5)}=\frac{\alpha \alpha_{Z}^{5}}{n^{3}}\left(\frac{89}{16}+\frac{8 \ln 2}{3}\right)=23.28 \frac{\alpha \alpha_{Z}^{5}}{\pi n^{3}} .
$$

This result, first obtained in 21], is hereby confirmed. It agrees with the coefficient 23.6(5) found numerically [22] for the ground state $n=1$. However, 22] finds a slight deviation from the scaling $1 / n^{3}$, contrary to [21] and us. This seems to indicate a poorer convergence of the numerical calculation than assessed in [22].

Two-loop self-interactions are significantly more chal- 
lenging. Numerically, they are known for the Lamb shift [23, 24], but for the $g$-factor only some classes of diagrams have been evaluated [25]. Analytical results are known for the full set of two-loop terms up to $\left(\frac{\alpha}{\pi}\right)^{2} \alpha_{Z}^{4}[26,27]$ and for some vacuum-polarization diagrams [28].

Effects of $\mathcal{O}\left(\left(\frac{\alpha}{\pi}\right)^{2} \alpha_{Z}^{5}\right)$ are becoming important for the determination of $m_{e}$. Since $g$ enters linearly in the master formula for $m_{e}$ 15, 16], the relative error in $g$ enters directly into the uncertainty of $m_{e}$. Numerically, $\left(\frac{\alpha}{\pi}\right)^{2} \alpha_{Z}^{5} /(g=2)$ is $4 \cdot 10^{-13}$ for carbon and $3 \cdot 10^{-11}$ for silicon. The current relative error in $m_{e}$ is $3 \cdot 10^{-11}$. Further improvements of $m_{e}$ hinge on the knowledge of the coefficient of $\left(\frac{\alpha}{\pi}\right)^{2} \alpha_{Z}^{5}$.

In the latest determination of $m_{e}$ [15, 16], that coefficient and $m_{e}$ were treated as two unknowns and were fitted to the two available results for $g$, in H-like carbon and silicon. (This illustrates opportunities offered by systems with various values of $Z$.) To this end, two-loop corrections were assumed to have the following expansion in $\alpha_{Z}$,

$$
\begin{aligned}
g^{(2)}= & \left(\frac{\alpha}{\pi}\right)^{2}\left[b_{00}\left(1+\frac{\alpha_{Z}^{2}}{6}\right)+\alpha_{Z}^{4}\left(b_{40}+b_{41} L\right)\right. \\
& \left.+\alpha_{Z}^{5} b_{50}+\alpha_{Z}^{6} b_{63} L^{3}+\ldots\right]
\end{aligned}
$$

The fit performed in [15, 16] resulted in $b_{50}(\mathrm{fit})=$ $-4.0(5.1)$. Here we directly determine contributions to $b_{50}$ beyond the already known vacuum-polarization effects $b_{50}^{\mathrm{VP}}[25,28]$. We write $b_{50}=b_{50}^{\mathrm{VP}}+\Delta b_{50}$ and set out to compute

$$
\Delta b_{50}=b_{50}^{\mathrm{SE}}+b_{50}^{\mathrm{LBL}}+b_{50}^{\mathrm{ML}},
$$

where the various terms originate with Feynman diagrams shown in Fig. 1.

Numerically the largest contribution to $b_{50}$ has been expected from $b_{50}^{\mathrm{SE}}$, generated by two-loop self-energy diagrams $\Sigma^{(2)}$. They correspond to the 19 diagrams contributing to Lamb shift at $\mathcal{O}\left(\alpha^{2} \alpha_{Z}^{5} m_{e}\right)$ [29 31] (see for example Fig. 2 in 31]). Each such diagram has five virtual electron propagators as well as external electron lines, all of which can interact with the magnetic field. Thus we must compute more than 100 diagrams, one example of which is Fig. 1(SE).

The $\alpha_{Z}^{5}$ corrections require at least two photons to be exchanged between the electron and the nucleus. The leading contribution arises when the momentum flowing in all three loops is of the order of electron mass (rather than for example $\mathcal{O}\left(\alpha_{Z} m_{e}\right)$ or $\mathcal{O}\left(\alpha_{Z}^{2} m_{e}\right)$, characteristic for so-called soft and ultrasoft effects). In this so-called hard region, exchanges of additional Coulomb photons are suppressed by $\alpha_{Z}$ so there is no need to resum them. The contribution is local from the perspective of the longdistance atomic scale set by the Bohr radius $\sim \frac{1}{\alpha_{Z} m_{e}}$ and can be modeled by a Dirac delta potential. Corrections to this approximation are expected to be powers and logarithms of the ratio of the Compton wavelength of the electron and the Bohr radius, $\mathcal{O}\left(\alpha_{Z}\right)$.

The contribution of such a short-distance potential to the $g$-factor equals $\frac{4}{m_{e}}$ times its expectation value (its contribution to the Lamb shift) [32]; the same result follows from virial relations 33]. Using the Lamb shift result [31], we find (for the ground state 1S; all our results can be generalized to $n \mathrm{~S}$ states by dividing by $n^{3}$ )

$$
g^{\mathrm{SE} ; \mathrm{Lamb}}=-9.83426(5) \alpha^{2} \alpha_{Z}^{5} .
$$

The uncertainty in this result comes from numerical errors in the master integrals computed in [31]. They affect all quantities we compute below using these three-loop integrals.

Self-energy diagrams influence $g$ also in two other ways. Their value depends on the energy of the electron, and that energy is shifted by the external magnetic field $\boldsymbol{B}$ by $\delta E=-g \frac{q}{2 m_{e}}\langle\boldsymbol{s}\rangle \cdot \boldsymbol{B}$. The resulting correction to $g$ is called $g_{3}$ in the notation of [21],

$$
g_{3}=\left.g \frac{\partial \Sigma}{\partial E}\right|_{E=m_{e}}=g_{3}^{(1)}+g_{3}^{(2)},
$$

where $g_{3}^{(1)}$ arises from the energy derivative of the oneloop self-energy multiplied by Schwinger's correction $\Delta g=\frac{\alpha}{\pi}$,

$$
g_{3}^{(1)}=\left(4 \ln 2-\frac{659}{64}\right) \frac{\alpha^{2}}{\pi} \alpha_{Z}^{5}
$$

and $g_{3}^{(2)}$ arises from the two-loop self-energy times the lowest order $g \rightarrow 2$.

Also the wave-function of the electron is modified by the magnetic field. This effect, together with the coupling of $\boldsymbol{B}$ inside self-energy diagrams like in Fig. 1(SE) provides the last SE correction $g_{4}$.

To evaluate the three-loop diagrams required for $g_{3}^{(2)}$ and $g_{4}$, we use the approach developed for the Lamb shift [31]. All three-loop diagrams are expressed in terms of 32 master integrals with the so-called Laporta algorithm [34, 35] implemented in the program FIRE [36]. Results for the master integrals and details of their computation can be found in 31]. Separately gauge-dependent, $g_{3}^{(2)}$ and $g_{4}$ add up to give a gauge-invariant result,

$$
g_{3}^{(2)}+g_{4}=12.816667(72) \alpha^{2} \alpha_{Z}^{5}
$$

The total SE correction is the sum of (5]7]8)

$$
g^{\mathrm{SE}}=0.58735(9) \alpha^{2} \alpha_{Z}^{5} .
$$

We note the remarkable cancelation of sizable partial contributions to $g^{\mathrm{SE}}$ in this sum.

The next correction comes from light-by-light scattering diagrams, one example of which is Fig. 1(LBL). They 
can be viewed as a term in the external-field expansion of self-energy diagrams with a vacuum-polarization insertion. In principle they can be calculated numerically along the lines of Ref. [25]. However, numerical treatment of virtual (unbound) electrons is challenging. Thus we include them here in the same manner as the SE corrections, again considering their Lamb-shift contribution and its energy dependence. Together with the direct magnetic field coupling effect, we find

$$
g^{\mathrm{LBL}}=-0.1724526(1) \alpha^{2} \alpha_{Z}^{5} .
$$

Diagrams with the external magnetic field coupling to the virtual electron loop are expected to be small [25]. In this class, we consider only the so-called magnetic loop contributions [37], examples of which are shown in Fig. 1(MLPH) and (MLVP). Their origin and evaluation differs from the diagrams we have discussed so far. Rather than modifying the response of the electron to a given external magnetic field, they represent the modification of the strength of the magnetic field caused by the electrostatic field of the nucleus.

There are three types of corrections to the leadingorder magnetic loop result, $\Delta g^{\mathrm{ML}}=\frac{7}{216} \alpha \alpha_{Z}^{5}$ found in [37]. Like in (77), there is a one-loop SE correction on the main electron line that provides

$$
g^{\mathrm{MLSE}}=\frac{7}{432} \frac{\alpha^{2}}{\pi} \alpha_{Z}^{5}
$$

Diagrams with a virtual photon inside the electron loop, see for example Fig. 1(MLPH), give

$$
g^{\mathrm{MLPH}}=\left(-\frac{7543}{16200}-\frac{303587}{10125 \pi}+\frac{92368}{2025 \pi} \ln 2\right) \alpha^{2} \alpha_{Z}^{5} .
$$

The third magnetic-loop contribution comes from inserting a second electron loop in one of the Coulomb-photon propagators, as in Fig. 1(MLVP),

$$
g^{\mathrm{MLVP}}=\left(\frac{628}{8505 \pi}-\frac{1}{54}\right) \alpha^{2} \alpha_{Z}^{5} .
$$

The total magnetic-loop correction is the sum of (11]12[13),

$$
g^{\mathrm{ML}}=0.064387 \ldots \alpha^{2} \alpha_{Z}^{5},
$$

a small effect, as expected [25]. Finally, we sum (9210[14) and get the total new correction,

$$
\Delta g^{(2,5)}=0.479287(90) \alpha^{2} \alpha_{Z}^{5},
$$

or, equivalently, a new contribution to $b_{50}$, defined in (4),

$$
\Delta b_{50}=4.7304(9) .
$$

The error estimate in this coefficient refers to the numerical uncertainty in the three-loop master integrals. We estimate the additional error due to the yet uncalculated VP diagrams at about 13 per cent of the value in (16), on the basis of the part we did evaluate, eq. (14). This error is presently negligible in comparison with unknown higher-order effects and we neglect it in the numerical analysis.

We note that the magnitude of the numerical coefficient in (15) is much smaller than in the previous order, see (2). This smallness may have complicated the experimental fit [15, 16]. Our final result (16) has opposite sign but similar magnitude to the fitted result [15, 16], $b_{50}$ (fit) $=-4.0(5.1)$. The difference between central values is 1.7 times the error assigned to the fit. Of course, the fit includes VP effects that we have not considered, but the known VP contributions are predominantly positive 25] and increase our discrepancy with the fit. On the other hand, the fit was done before the $\mathcal{O}\left(\alpha_{Z}^{4}\right) \mathrm{LBL}$ effect was computed [27]; including it may improve the agreement [38].

How does the new correction in eq. (15) influence the determination of the electron mass? In case of the carbon ion, the relative size of the change in $g$ is $2 \cdot 10^{-12}$. The atomic electron mass is directly proportional to $g$ so this change increases $m_{e}$ by the same relative amount, well below the current error of $3 \cdot 10^{-11}$ [15, [16]; in absolute terms, by about $10^{-15}$ atomic mass units.

This stability of the electron mass should not be taken for granted. Partial results such as (8) correspond to relative shifts as large as $5 \cdot 10^{-11}$, larger than the current uncertainty in $m_{e}$. They have, however, been canceled by other effects such as $g^{\text {SE;Lamb in (5). }}$.

In Table \ we summarize results for hydrogen-like helium, carbon and silicon ions. The first line corresponds to Breit's formula (11). The second line includes additionally all known corrections preceding this work, as given in [16]: finite nuclear size [39]; one-loop QED corrections obtained by combining numerical and analytical computations, 22, 26, 37, 40 48]; two-loop QED corrections evaluated up to order $\alpha_{Z}^{4}$ [27, 46, 47]; higher order terms for diagrams with vacuum polarization insertions [25]; three and more QED loops up to order $\alpha_{Z}^{2}$ [3]; recoil and radiative recoil [49 51] (see also [52]); nuclear polarizability [53] and susceptibility [54]; as well as leading weak [55] and hadronic effects [56, 57].

For carbon and silicon the theoretical error in the second row of Table \is dominated by the finite nuclear size effects, whose error is respectively $\delta g=7 \times 10^{-13}$ and $\delta g=31 \times 10^{-12}$. For helium, the main uncertainty comes from the poorly known one-loop corrections extrapolated from numerical computation.

We estimate the remaining error due to still missing higher order terms by the leading logarithm $\left(\frac{\alpha}{\pi}\right)^{2} \alpha_{Z}^{6} \ln ^{3} \alpha_{Z}^{-2}$. In carbon and silicon, this logarithmic term exceeds other uncertainties as well as our correction. It also exceeds the uncertainty estimated in [16], where for Si ion the error was $139 \cdot 10^{-12}$. 
It may seem ironic that after all the effort of computing $\Delta b_{50}$ our predictions for $g$ have larger uncertainties than before our work. We believe however that this conservative treatment of unknown higher orders is necessary.

In [15], $b_{50}$ was fitted and the higher-order terms were neglected. This procedure raises some concerns since the next term, $b_{63}$, is logarithmically enhanced. For small $Z$, that enhancement may overshadow the $b_{50}$ term. Yet, with experimental results available only for carbon and silicon it was impossible to constrain that higher-order term. Now that the $b_{50}$ term is at hand, one can fit the logarithmically enhanced term $b_{63}$ while neglecting parametrically suppressed terms starting from $b_{62}$.

In this way, our result will help derive full benefit from future measurements of $g$-factors, an effort we admire and encourage.

\section{ACKNOWLEDGMENTS}

We thank Zoltan Harman for helpful discussions. We thank Jorge Mondéjar for collaboration in the early stages of this project. The loop diagrams were calculated with FORM [58]. This research was supported by Science and Engineering Research Canada (NSERC) and by the Munich Institute for Astro- and Particle Physics (MIAPP) of the DFG cluster of excellence "Origin and Structure of the Universe."

TABLE I. Bound electron $g$-factor for helium, carbon and silicon ions. The error related to missing higher order contributions is estimated by $\left(\frac{\alpha}{\pi}\right)^{2} \alpha_{Z}^{6} \ln ^{3} \alpha_{Z}^{-2}$.

\begin{tabular}{lcccc}
\hline \hline Contribution & ${ }^{4} \mathrm{He}^{+}$ & ${ }^{12} \mathrm{C}^{5+}$ & ${ }^{28} \mathrm{Si}^{13+}$ & \\
\hline Dirac/Breit value & $1.99985798882537(6)$ & $1.9987213543921(6)$ & $1.993023571557(3)$ & {$[20]$} \\
+ other known corrections & $2.00217740671141(55)$ & $2.0010415901686(12)$ & $1.995348957825(39)$ & {$[16,22]^{\mathrm{a}}$} \\
& & & & \\
$g^{\mathrm{SE}}$ & 0.00000000000002 & 0.0000000000050 & 0.000000000348 & (this work) \\
$g^{\mathrm{LBL}}$ & -0.00000000000001 & -0.0000000000015 & -0.000000000102 & (this work) \\
$g^{\mathrm{ML}}$ & 0.00000000000000 & 0.0000000000006 & 0.000000000038 & (this work) \\
\hline$\left(\frac{\alpha}{\pi}\right)^{2} \alpha_{Z}^{6} \ln ^{3} \alpha_{Z}^{-2}$ & $0.00000000000000(3)$ & $0.0000000000000(93)$ & $0.000000000000(583)$ & \\
\hline Total & $2.00217740671142(55)$ & $2.0010415901727(94)$ & $1.995348958109(584)$ \\
\hline \hline
\end{tabular}

a The only change with respect to [16] is that we used the new evaluation of [22] for the one-loop corrections.

[1] P. J. Mohr, D. B. Newell, and B. N. Taylor, Rev. Mod. Phys. 88, 035009 (2016), arXiv:1507.07956 [physics.atom-ph].

[2] J. S. Schwinger, Phys. Rev. 73, 416 (1948)

[3] T. Aoyama, M. Hayakawa, T. Kinoshita, and M. Nio, Phys. Rev. Lett. 109, 111807 (2012) arXiv:1205.5368 [hep-ph]

[4] S. Laporta, Phys. Lett. B772, 232 (2017), arXiv:1704.06996 [hep-ph]

[5] G. Gabrielse, Adv. Ser. Direct. High Energy Phys. 20, 157

[6] H. Häffner, T. Beier, N. Hermanspahn, H. J. Kluge, W. Quint, S. Stahl, J. Verdú, and G. Werth, Phys. Rev. Lett. 85, 5308 (2000)

[7] T. Beier, H. Haffner, N. Hermanspahn, S. G. Karshenboim, H. J. Kluge, W. Quint, S. Stahl, J. Verdu, and G. Werth, Phys. Rev. Lett. 88, 011603 (2001).

[8] F. Köhler, S. Sturm, A. Kracke, G. Werth, W. Quint, and K. Blaum, J. Phys. B48, 144032 (2015) arXiv:1604.04380 [physics.atom-ph].

[9] S. Sturm, A. Wagner, M. Kretzschmar, W. Quint, G. Werth, and K. Blaum, Phys. Rev. A87, 030501 (2013)
[10] S. Sturm, M. Vogel, F. Köhler-Langes, W. Quint, K. Blaum, and G. Werth, Atoms 5, 4 (2017).

[11] A. Wagner, S. Sturm, F. Köhler, D. A. Glazov, A. V. Volotka, G. Plunien, W. Quint, G. Werth, V. M. Shabaev, and K. Blaum, Phys. Rev. Lett. 110, 033003 (2013), arXiv:1406.6501 [physics.atom-ph].

[12] M. Vogel and W. Quint, Springer Tracts Mod. Phys. 256, 73 (2014).

[13] R. Soria Orts, J. R. Crespo López-Urrutia, H. Bruhns, A. J. González Martínez, Z. Harman, U. D. Jentschura, C. H. Keitel, A. Lapierre, H. Tawara, ). I. Tupitsyn, J. Ullrich, and A. V. Volotka, Phys. Rev. A76, 052501 (2007).

[14] M. S. Safronova, D. Budker, D. DeMille, D. F. J. Kimball, A. Derevianko, and C. W. Clark, "Search for New Physics with Atoms and Molecules," (2017), arXiv:1710.01833.

[15] S. Sturm, F. Köhler, J. Zatorski, A. Wagner, Z. Harman, G. Werth, W. Quint, C. H. Keitel, and K. Blaum, Nature 506, 467 (2014).

[16] J. Zatorski, B. Sikora, S. G. Karshenboim, S. Sturm, F. Köhler-Langes, K. Blaum, C. H. Keitel, and Z. Harman, Phys. Rev. A96, 012502 (2017), arXiv:1703.10649 [physics.atom-ph], 
[17] V. M. Shabaev, D. A. Glazov, N. S. Oreshkina, A. V. Volotka, G. Plunien, H.-J. Kluge, and W. Quint, Phys. Rev. Lett. 96, 253002 (2006).

[18] V. A. Yerokhin, E. Berseneva, Z. Harman, I. I. Tupitsyn, and C. H. Keitel, Phys. Rev. Lett. 116, 100801 (2016).

[19] V. A. Yerokhin, E. Berseneva, Z. Harman, I. I. Tupitsyn, and C. H. Keitel, Phys. Rev. A94, 022502 (2016) arXiv:1606.08620 [physics.atom-ph].

[20] G. Breit, Nature 122, 649 (1928).

[21] K. Pachucki and M. Puchalski, $\quad$ Phys. Rev. A96, 032503 (2017) arXiv:1707.08518 [physics.atom-ph].

[22] V. A. Yerokhin and Z. Harman, $\quad$ Phys. Rev. A95, 060501 (2017), arXiv:1704.08080 [physics.atom-ph].

[23] V. A. Yerokhin, P. Indelicato, and V. M. Shabaev, Phys. Rev. Lett. 91, 073001 (2003), arXiv:hep-ph/0302268 [hep-ph].

[24] V. A. Yerokhin, P. Indelicato, and V. M. Shabaev, Phys. Rev. A71, 040101 (2005), hep-ph/0411348

[25] V. A. Yerokhin and Z. Harman, Phys. Rev. A88, 042502 (2013).

[26] K. Pachucki, A. Czarnecki, U. D. Jentschura, and V. A. Yerokhin, Phys. Rev. A72, 022108 (2005), physics/0506227.

[27] A. Czarnecki and R. Szafron, Phys. Rev. A94, 060501 (2016), arXiv:1611.04875 [physics.atom-ph].

[28] U. D. Jentschura, Phys. Rev. A79, 044501 (2009).

[29] K. Pachucki, Phys. Rev. Lett. 72, 3154 (1994).

[30] M. I. Eides and V. A. Shelyuto, Phys. Rev. A52, 954 (1995), hep-ph/9501303

[31] M. Dowling, J. Mondéjar, J. H. Piclum, and A. Czarnecki, Phys. Rev. A81, 022509 (2010), arXiv:0911.4078 [hep-ph]

[32] S. G. Karshenboim, Phys. Lett. A266, 380 (2000).

[33] V. M. Shabaev, in Precision Physics of Simple Atomic Systems, edited by S. G. Karshenboim and V. B. Smirnov (Springer, Berlin, 2003) p. 97, physics/0211087.

[34] S. Laporta and E. Remiddi, Phys. Lett. B379, 283 (1996), hep-ph/9602417

[35] S. Laporta, Int. J. Mod. Phys. A15, 5087 (2000), hep-ph/0102033.

[36] A. V. Smirnov, Comput. Phys. Commun. 189, 182 (2014) arXiv:1408.2372 [hep-ph]

[37] S. G. Karshenboim and A. I. Mil- stein, arXiv:hep-ph/0210069 [hep-ph].

[38] Z. Harman, private communication (2017).

[39] J. Zatorski, N. S. Oreshkina, C. H. Keitel, and Z. Harman, Phys. Rev. Lett. 108, 063005 (2012).

[40] H. Grotch, Phys. Rev. Lett. 24, 39 (1970)

[41] M. I. Eides and H. Grotch, Ann. Phys. 260, 191 (1997).

[42] A. Czarnecki, K. Melnikov, and A. Yelkhovsky, Phys. Rev. A63, 012509 (2000), hep-ph/0007217.

[43] S. G. Karshenboim, V. G. Ivanov, and V. M. Shabaev, J. Exp. Theor. Phys. 93, 477 (2001), [Zh. Eksp. Teor. Fiz.120,no.3,546(2001)].

[44] V. A. Yerokhin, P. Indelicato, and V. M. Shabaev, Phys. Rev. Lett. 89, 143001 (2002).

[45] R. N. Lee, A. I. Milstein, I. S. Terekhov, and S. G. Karshenboim, Phys. Rev. A71, 052501 (2005), arXiv:hep-ph/0412026 [hep-ph].

[46] V. A. Yerokhin, P. Indelicato, and V. M. Shabaev, Phys. Rev. A69, 052503 (2004)

[47] K. Pachucki, U. D. Jentschura, and V. A. Yerokhin, Phys. Rev. Lett. 93, 150401 (2004), erratum Phys. Rev. Lett. 94, 229902(E) (2005), hep-ph/0411084.

[48] V. Yerokhin, C. Keitel, and Z. Harman, Journal of Physics B: Atomic, Molecular and Optical Physics 46, 245002 (2013).

[49] V. M. Shabaev and V. A. Yerokhin, Phys. Rev. Lett. 88, 091801 (2002).

[50] T. Beier, Phys. Rep. 339, 79 (2000).

[51] K. Pachucki, Phys. Rev. A78, 012504 (2008).

[52] D. A. Glazov, A. V. Volotka, V. M. Shabaev, and G. Plunien, Springer Tracts Mod. Phys. 256, 137 (2014)

[53] A. V. Nefiodov, G. Plunien, and G. Soff, Phys. Rev. Lett. 89, 081802 (2002).

[54] U. D. Jentschura, A. Czarnecki, K. Pachucki, and V. A. Yerokhin, Int. J. Mass Spectrometry 251, 102 (2006), arXiv:physics/0510049 [physics]

[55] A. Czarnecki, B. Krause, and W. J. Marciano, Phys. Rev. Lett. 76, 3267 (1996) arXiv:hep-ph/9512369 [hep-ph]

[56] D. Nomura and T. Teubner, Nucl. Phys. B867, 236 (2013) arXiv:1208.4194 [hep-ph],

[57] A. Kurz, T. Liu, P. Marquard, and M. Steinhauser, Phys. Lett. B734, 144 (2014), arXiv:1403.6400 [hep-ph].

[58] J. A. M. Vermaseren, "New features of FORM," (2000), math-ph/0010025. 